\title{
Projecting emotions from artworks to maps using neural style transfer
}

\author{
Edyta P. Bogucka*, Liqiu Meng \\ Chair of Cartography, Technical University of Munich, e.p.bogucka@tum.de, liqiu.meng@tum.de \\ * Corresponding author
}

\begin{abstract}
Recent advances in deep learning have facilitated the exchange of styles and textures between input images to create unique synthesised outputs. This paper assesses the applicability of neural style transfer to cartography and evaluates to what degree emotions attached to input images can be preserved in maps co-created by human and algorithm. As a source of emotions we utilized personal paintings created during a workshop with international artists at the School of Machines, Making \& Make-Believe in August 2018. The neural style transfer was used as a tool to transfer the characteristics of the artworks onto the map. Differences in emotion perception between human-made textures and generated maps were assessed with an online survey completed by 1187 users. The results confirmed that emotional descriptions remain the same before and after the procedure of neural style transfer. The users perceived artificially generated maps as interesting and visually pleasing artefacts. Artworks with variety of line, point and surface depictions were the most suitable algorithm inputs and achieved better visual results in representing the map content. After analysing the neural style transfer technique and identifying its limitations for cartographic style and map content, we conclude with plausible directions for future research.
\end{abstract}

Keywords: neural style transfer, art, style extraction, emotional mapping

\section{Introduction}

In the era of ubiquitous sensors, social media and Volunteered Geographic Information, we are experiencing the renaissance of mapping. One of the opportunities to make sense of geospatial big data is to develop novel visualization approaches by encouraging creative contributions (Robinson et al., 2017). Cartographers might therefore re-think their role in two directions (Kent, 2017). Firstly, by enhancing designer skills with artistic styles (Sidonie, 2012); secondly, by being more open to experimental tools for visual expression. The creative community raises currently the question on how the concepts of deep learning and artificial intelligence might influence future artistic production. Open datasets and algorithms used in machine learning research are immediately taken up by artists to create playful, provoking, border-pushing art. However, it is still not common to utilize those tools to address aesthetic and functional aspects of map design.

Recent advances in deep learning allow exchanging styles and textures between images, what might enhance cartographic practice in multiple ways. One can freely collect the style inputs such as photographs, maps or selfmade drawings. This self-curated collection of visual inspirations expresses and sharpens a personal definition of cartographic style and forms a basis for personal visual narratives (Thudt et al., 2017). In the next steps maps can be re-used as intermediate spatial frames to be filled with various textures. Different cartographic design of input maps will, therefore, lead to unique, synthesized mapbased artworks. The cartographer can control and fine-tune multiple algorithm parameters, as several implementations (Johnson, 2015, Smith, 2016) allow colour control or merging multiple style sources. This paper presents a workflow of utilizing neural style transfer as a tool for emotional mapping.

The remainder of the paper is organized into six sections. In the following section we reflect on the concept of neural style transfer and list a few applications of this technique in cartography. The methodology of projecting emotions from artworks to maps is further explained in section 3 . Section 4 introduces the case study of Berlin and evaluation of human-made textures and machine-made maps with the online survey. Section 5 summarizes the results of this work. It provides users' emotional impressions and insights into this new form of mapping. The implications of the neural style transfer in cartographic design are further discussed in section 6 . The final section 7 reflects on the further usage of this technique in cartography.

\section{State of the art}

The innovating implementation of style transfer as an image stylisation technique was proposed by Gatys et al. in 2015. The method utilizes two input images $x_{c}$ and $x_{s}$ to synthesize a third image $x_{n}$, which shares a semantic content of the first image $\mathrm{x}_{\mathrm{c}}$ and the style of the second $\mathrm{x}_{\mathrm{s}}$. The concepts of style and content have double interpretation, meaning both image statistics and creative way the artworks are made (Gombrich, 1968). The first generation of style transfer algorithms is already 
established and often used in advanced photo-editing applications such as Prisma or Dreamscope.

The subsequent implementations of style transfer perceive it rather as a general cross-domain adaptation problem (Isola et al., 2017, Zhu et al., 2017). With the rise of generative adversarial networks (GANs), researchers were able to generalize the style over multiple paired and unpaired images and translate it from one image to another. This cross-domain style transfer possibility was creatively explored by artists, who delivered grasping examples of turning sketches into imaginary landscapes (Karacan et al., 2018) or own faces into historical portraits (Klingemann, 2018).

The potential of this technique has not been explored yet in cartographic applications. The online platform ml5js for web-based machine learning shared a pre-trained GAN model to synthesize maps from aerial imagery (2018). Two other projects focused on training models to extract the unique character of the cities. OpenDot Lab used current maps and aerial images of Milan, Venice and Los Angeles to transfer the urban structures such as paved roads from one city to another (Kogan et al., 2016). Clark (2017) reimagined ancient cities of Jerusalem, Babylon and London by turning historical hand-drawn city maps into modern satellite views.

As people are not yet exposed to artificially styled maps, there is a strong need to discover what are their attitudes towards this experimental form of cartography. Field and Demaj (2012) perceive machine-made maps as not capable to evoke emotional responses from the map readers. However, they acknowledge that some of the most evocative maps utilize a completely new approach to a mapping task. Emotional associations may be caused by a map itself, but also by data visualized in the mapping process. The main sources of emotional datasets are human bodies itself (Nold, 2009), social media or VGI (Griffin and McQuoid, 2012). Not aiming at evoking impact on users, this paper utilizes art as a source of emotions and places them in the spatial context with the use of neural style transfer.

\section{Methodology}

For this work we apply a neural style transfer model (Gatys et al., 2016) that has been widely adopted by the creative machine learning community. It is based on the Convolutional Neural Networks (CNNs) that are used for computer vision and machine learning tasks to extract complex hierarchical representations of images. CNNs are arranged as a sequence of cascaded layers, which extract increasingly complex characteristics from processed images. The first CNN layers typically extract low-level representations, such as basic shapes or textures, while deeper layers encode higher-level representations, such as global structures and complex shape of objects. With neural style transfer (Gatys et al., 2016), characteristics for content $F_{c}$ and style $F_{s}$ are extracted separately from two source images (Figure 1). The first one represents the artwork-style, the second conveys the overall structure of the scene-content. This is accomplished via a dual objective function. The style-objective encourages the network to extract characteristics similar to the presented artwork over multiple hierarchical network layers, while the content-objective encourages deeper high-level representations to be similar to the content image. Once the model is trained on a specific style image, a variety of content images can be processed, making this a versatile tool for the extraction of complex patterns in style and texture. In our approach style-to-map exchange is seen as a transfer of emotions associated with one form of abstraction (artwork) to the abstract representation of the reality (map).

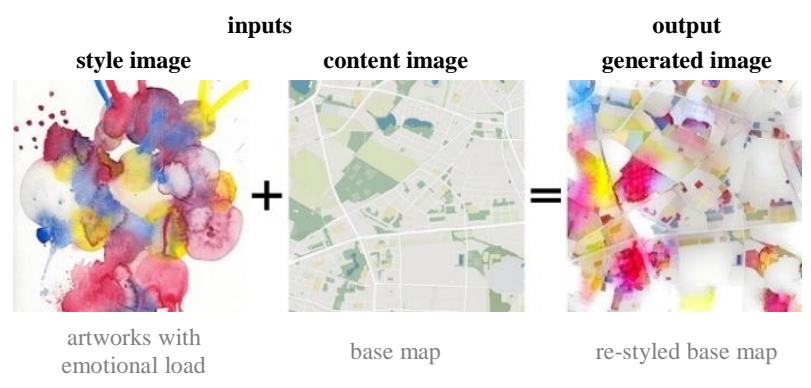

Figure 1. Synthesizing artistic maps from personalized artworks and base maps using style transfer technique.

\section{Experiments}

\subsection{Creating inputs}

The content map input was created in Mapbox Studio and consisted of roads, buildings and land cover classes represented in light colour scheme (Figure 2). No labels were used to annotate objects on the map, as within the style transfer they could have added noise to the output image. The handmade style inputs were collected at the School of Machines, Making \& Make-Believe in Berlin during the Autonomous Generative Spirit Programme in August 2018. The participants were asked to reflect on their emotions about Berlin and to visualize them in the painting process, focussing on conveying the unique style of their feelings by the choice of colours, lines, and painted structures. The participants were using the same painting supplies - watercolours and thick watercolour paper. The task was not time-limited. Based on their feelings, 16 textures in both abstract and realistic styles were created and scanned. The last input image combined all personal paintings together to express a collaborative, emotional image of the city.

\subsection{Generating outputs}

The personal emotional images were projected to the base map using the neural style transfer. The source code with algorithm implementation (Johnson, 2015) was forked from GitHub repository and deployed on the virtual machine in Paperspace. The style and content weights were set as 0.5 , which means that each input had the equal impact on the final image. The results of the transfer are presented on the Figure 3 and are available in high resolution at www.edytabogucka.de. 


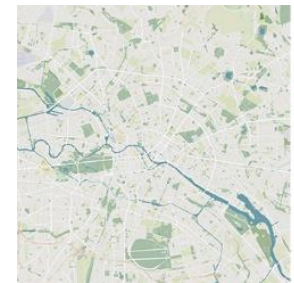

basemap

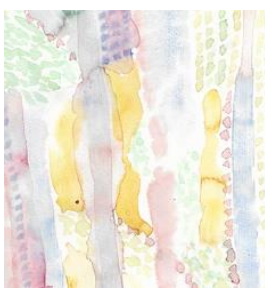

$\mathrm{H}$

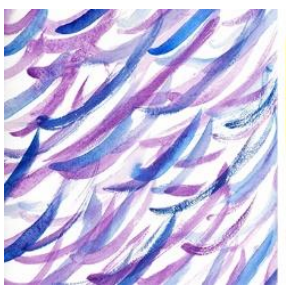

RO

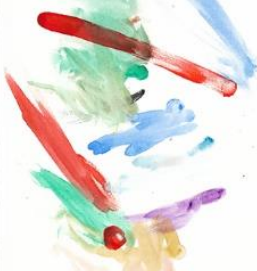

A

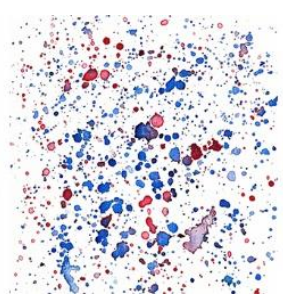

AN

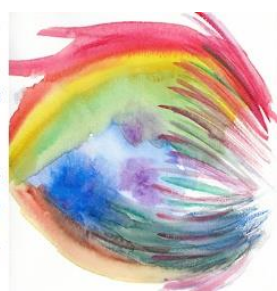

$\mathrm{E}$

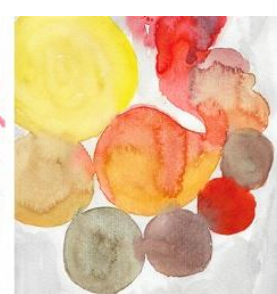

$\mathrm{F}$

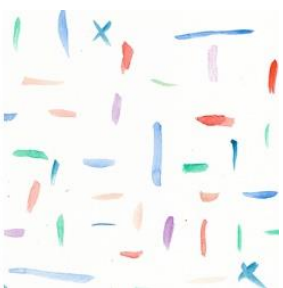

G

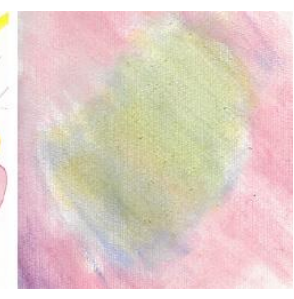

$\mathrm{R}$

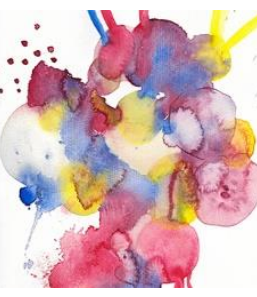

$\mathrm{N}$

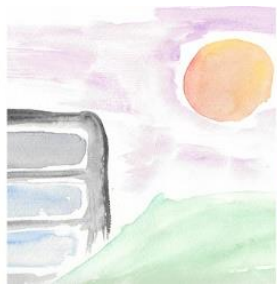

J

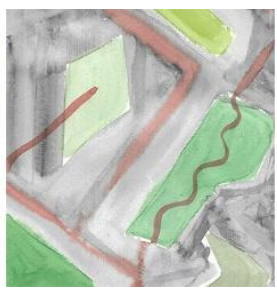

K

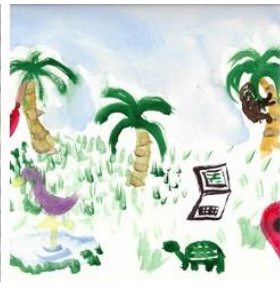

M

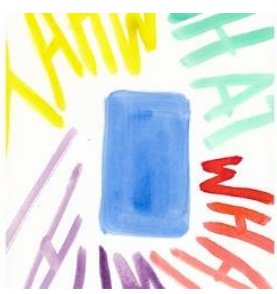

S

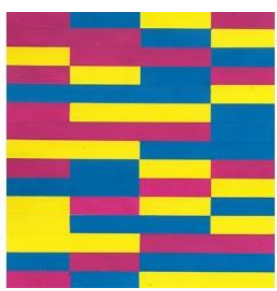

$\mathrm{T}$

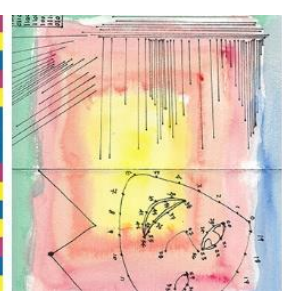

TR

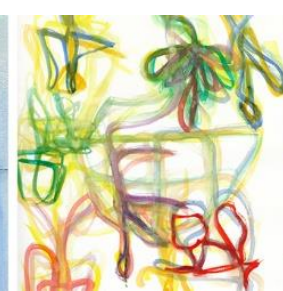

Z

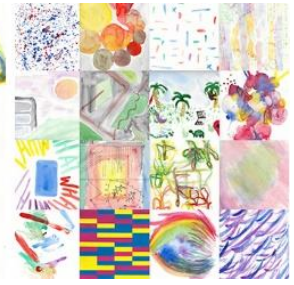

C (joint styles)

Figure 2. Style input images - personal watercolour textures and digitally joined group texture.

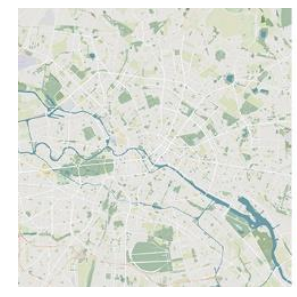

basemap

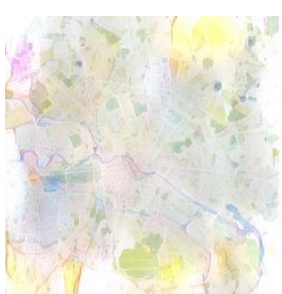

$\mathrm{H}$

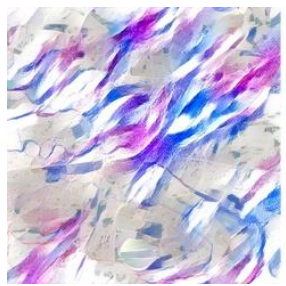

RO

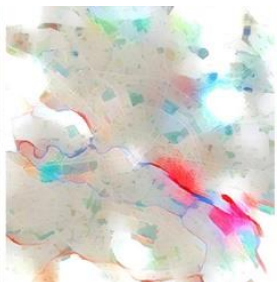

A

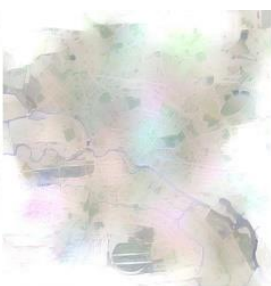

J

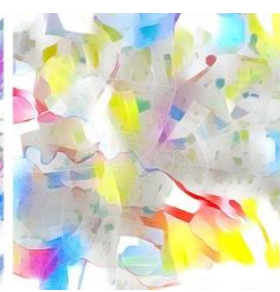

$\mathrm{S}$

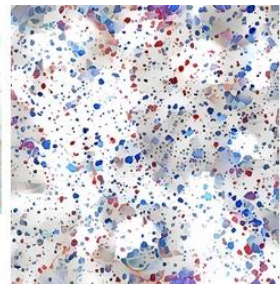

AN

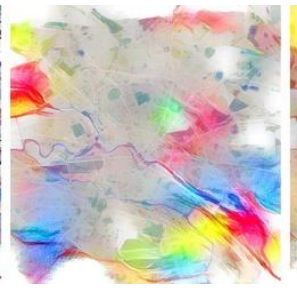

E

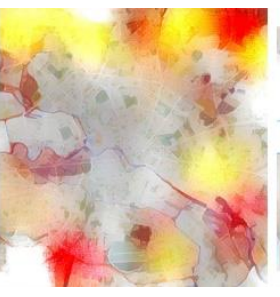

F

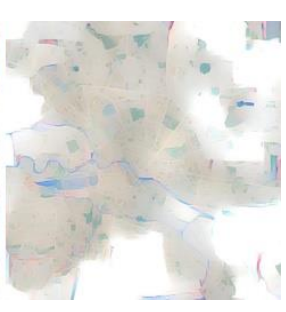

G

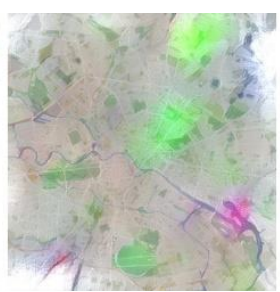

K

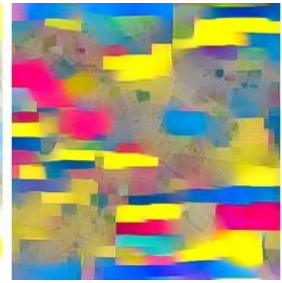

$\mathrm{T}$

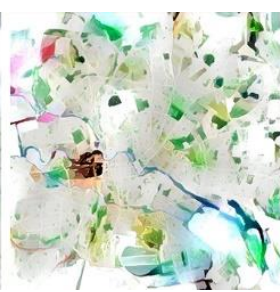

M

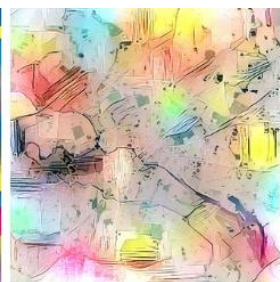

TR

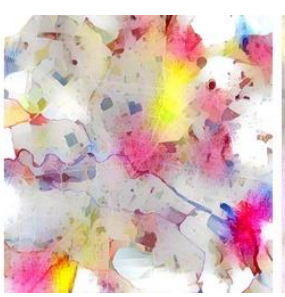

$\mathrm{N}$

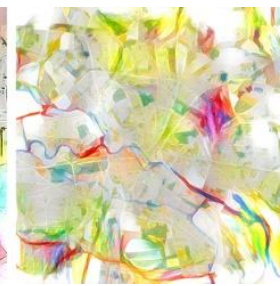

Z

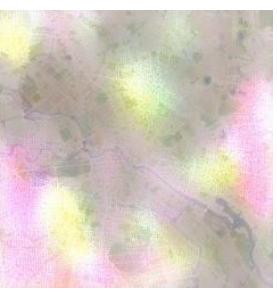

R

Figure 3. Emotional maps of Berlin as generated from the human-made paintings with the style transfer algorithm. 


\subsection{Survey}

The goal of the survey was to evaluate the ability of neural style transfer to maintain emotional associations. Users were asked six questions to one randomly assigned image being either a painted texture (17 items) or a map (17 items). Questions regarding emotional perception drew from emotion categorization models of hourglass (Cambria et al., 2012) and emotion wheel (Plutchik, 1980). Emotion pair in each category represents the opposite feelings which can not be experienced at the same time (Table 1). To assess the magnitude of the feeling, sevenpoint Likert-scale was set with a neutral value in the middle and maximum values at the ends. Closed-response, single-choice questions minimized the survey time in order to get quick emotional impressions.

\begin{tabular}{|l|l|l|l|l|}
\hline Setting & Q1 & Q2 & Q3 & Q4 \\
\hline $\begin{array}{l}\text { Emotion } \\
\text { category }\end{array}$ & Pleasantness & Attention & Aptitude & Sensitivity \\
\hline Emotion pair & $\begin{array}{l}\text { sadness - } \\
\text { joy }\end{array}$ & $\begin{array}{l}\text { distraction - } \\
\text { interest }\end{array}$ & $\begin{array}{l}\text { disgust - } \\
\text { trust }\end{array}$ & $\begin{array}{l}\text { anger - } \\
\text { fear }\end{array}$ \\
\hline $\begin{array}{l}\text { Is this } \\
\text { image... ? }\end{array}$ & $\begin{array}{l}\text { sad or } \\
\text { happy? }\end{array}$ & $\begin{array}{l}\text { distracting } \\
\text { or } \\
\text { interesting? }\end{array}$ & $\begin{array}{l}\text { disgusting } \\
\text { or } \\
\text { trustful? }\end{array}$ & $\begin{array}{l}\text { angry or } \\
\text { scary? }\end{array}$ \\
\hline $\begin{array}{l}\text { Response } \\
\text { format }\end{array}$ & $\begin{array}{l}\text { Single choice, Likert scale ranking, e.g.: } \\
\text { extremely sad - sad - slightly sad - neutral - slightly } \\
\text { happy - happy - extremely happy }\end{array}$ \\
\hline
\end{tabular}

Table 1. Emotion categories evaluated in the questionnaire.

Once the survey was started, participants were asked to observe a single image and complete the tasks. The image was either a raw texture or a styled map. Users interacting with the generated map were then asked to write down what do they see $\left(\mathrm{Q}^{*}\right)$. In the next step, participants were describing the emotional load of the image (Q1-Q4). At the end of the questionnaire, users completed a demographics section about their gender (Q5) and age (Q6).

Test surveys were distributed among the participants with the use of the Amazon's crowdsourcing platform Mechanical Turk, which defines the participation in the test as a Human Intelligence Task (HIT). Minimum participants' qualifications were specified in the settings of HIT. The number of surveys taken by the user so far should be greater than 100 and approval rate (\%) for survey answers should be greater than 95 . The surveys were open for data collection for 14 days and the suggested time to complete the tasks was approx. three minutes.

\section{Results}

The study was completed by 1187 users in the age between 18-64. The test sample ranged between $30-38$ participants per image. To determine if there were differences in emotion perception between textures and maps, independent samples were assessed with the Mann Whitney U test. Results for the analysis are summarized in the Appendix Table 2 and in the descriptive statistics (Figure 4).
The results confirmed the expectation that emotions may remain the same before and after style transfer procedure. For 10 pairs of artwork and map, test has shown no significant difference in answers to all questions at alpha $=$ '0.05'. For the remaining 7 artworks significant differences occurred in one or two questions, which means that input and output images conveyed different emotional load in those categories.

In the pleasantness category (Q1) images were mostly rated as happy (mode value of $53 \%$ of all images) or slightly happy (mode value of $44 \%$ of all images). A significant difference at alpha $=$ ' 0.01 ' was found for the textures $\mathrm{E}$ and $\mathrm{N}$. Map created from the texture $\mathrm{N}$ was assessed "happier" than the original input. Textures E and $\mathrm{G}$ were scored less happy when transferred to a map. Based on the average values, the happiest maps were generated from textures $\mathrm{S}, \mathrm{N}$ and $\mathrm{T}$.

The distribution of answers to the Q2 (attention) was more unified in comparison to $\mathrm{Q} 1$. The images raised the emotions of interest (mode and mean value of $47 \%$ of all images) or slight interest (mode and mean value of $50 \%$ of all images). Artworks $\mathrm{K}$ and $\mathrm{S}$ statistically gained more interest after style transfer procedure. Maps evoking the highest interest emotions were generated from textures $\mathrm{S}$, $\mathrm{M}$ and RO.

The aptitude emotions (Q3) were grouped around slightly trustful (mode and mean value of $59 \%$ of all images) and trustful values (mode and mean value of $35 \%$ of all images). Textures $\mathrm{E}$ and $\mathrm{M}$ gained less trust when projected on the map, whereas texture $\mathrm{N}$ got more reliable. In the last category (sensitivity) $85 \%$ of images evoked neither fear nor anger. Artworks $\mathrm{F}$ and $\mathrm{K}$ statistically appeared less scary after the style transfer (change from mean value of "slightly scary" to "neutral"), contrary to the texture $\mathrm{M}$.

The descriptive statistics also present the input feelings of 11 artists - authors of the paintings. It was observed that survey participants have not encoded all artists' intentions in the same way. Textures $\mathrm{A}$ and $\mathrm{H}$ were created to convey sadness, but were assessed by the users as happy. Similarly, paintings $\mathrm{TR}$ and $\mathrm{H}$ were not recognized as distracting.

Users assessing artificially generated maps also provided comments on the content of the images. Their answers were then grouped into four categories - map, art, colour, or other. The highest map recognizability was achieved by applying texture $\mathrm{K}-69 \%$ of respondents used a keyword "map" to describe the image content. Similarly recognizable were maps based on texture $\mathrm{J}(65 \%)$ and $\mathrm{A}$ $(62 \%)$. Image generated from texture AN has not been associated with a map ( $0 \%$ of answers), whereas for all other images this word appeared minimum once. The word "art" has been used the most often for map-based images from textures S (27\%), N (27\%), E and M (24\%). As the most colourful users found maps created from artworks $\mathrm{C}$ $(46 \%)$, AN (43\%) and S (38\%). 

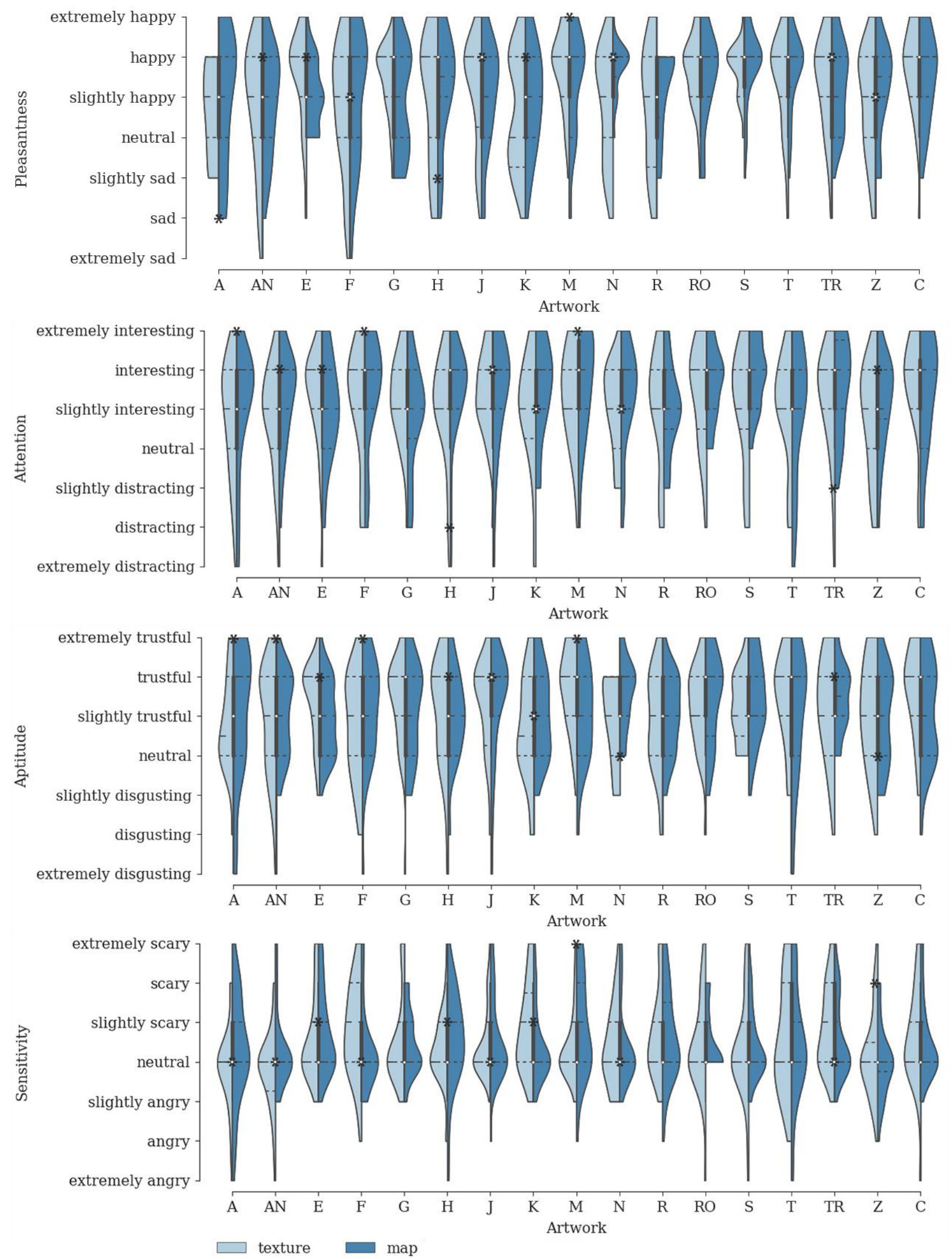

Figure 4. Descriptive statistics by emotion category, image type and artwork. On plots median is shown as a white dot and quartiles as horizontal lines. Original emotions of artists are marked with an asterisk. Thickness of the violin plot indicate the number of observations. 


\section{Discussions}

The achieved results encourage further use of the style transfer techniques in experimental mapping. It was expected that resulting maps would be rather described as disgusting and mistrustful in comparison to input images. This suggests that the effect of unwarranted trust users put in maps they interact with (Tyner, 1982) is also present in artificially generated maps. All textures and maps evoked positive emotions of happiness and interest. However, the artwork dataset was skewed towards positive emotion categories, as anger and fear were not represented on the collected paintings. In the next step the sample of input textures should be extended by adding more diverse inputs, e.g. from famous artworks which are widely known for conveying anger or disgust. Assigning and evaluating textures for all spectrum of emotions is crucial, as the most commonly mapped emotions in the cities are fear and discomfort (Griffin and McQuoid, 2012).

The main critique point of style transfer is an unintended randomness, which causes inconsistencies in style and changes the map content. The following negative effects were observed in the artificially generated maps: symbolizing different objects with the same colours, presence of new colours not used in the input textures (Figure 5a), exaggerating the texture of watercolour paper medium (Figure $5 \mathrm{~b}$ ) and visual similarity of outputs given different inputs (Figure 5c, 5f). The semantic content of maps was influenced by adding new, non-existing geographical objects (multiple parallel roads on Figure 5d) or removing objects (absence of an old airport on Figure $5 \mathrm{f}$ in comparison to Figure $5 \mathrm{c}$ ).

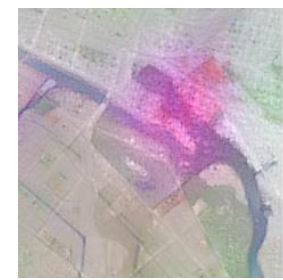

a) $\mathrm{K}$

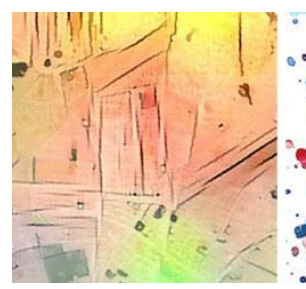

d) TR

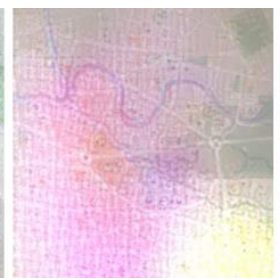

b) $\mathrm{R}$

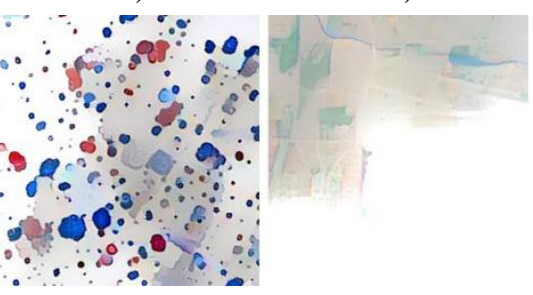

e) AN

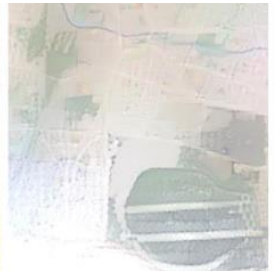

c) $\mathbf{J}$
Figure 5. Negative implications of style transfer in cartographic style and map content.

When treated as an experimental tool, style transfer might help to creatively re-think the way how cities are mapped by machines. One of the challenges is to understand why some artwork styles emphasize urban structures, while other not. Based on the results, scattered and dotted textures (AN) and artworks with plain colour surfaces (R) may not be suitable for this purpose. The best visual results were achieved by the textures with variety of line, point and surface representations. Especially interesting is artwork TR, which utilizes a mixed technique of watercolour and pen. Thin black lines have been reused by the algorithm to outline streets and buildings. The original paper (Gatys et al., 2015) did not recommend using multiple artworks with different styles, as the algorithm would not be able to generalize the "average" style over them. Contrary to this suggestion, the results show that the most colourful city map was created from the joint texture. The combination of artistic representations with base map produces a hybrid map, in which the colours and forms might be disconnected from the places they represent. These negative effects can be partially addressed by adjusting algorithm parameters such as style-content ratio or using content and style masks. The network will then utilize only a subset of the style representation and transfer it to the selected part of the map. These modifications give more control over the cartographic design process and make such experimental maps more meaningful.

The created abstract maps of the city are highly subjective, as they merge artworks, personal perceptions of the space and map representations. It cannot be ensured that generated map will evoke the same or any aesthetic pleasure in map viewer, although this assumption is common in image processing and computer vision (You et al., 2016, Machajdik and Hanbury, 2010). Instead, artificially styled maps may be useful to express personal narratives and tell unique stories - they are vivid and valid for single person or an artist's group in a particular lifetime and location.

\section{Conclusions}

Style transfer may help to extract visual characteristics of data, and can thus be utilized with both the big data (generating a style over multiple images) and artisanal data (purposefully sampled small datasets). Further developments in deep learning open new possibilities to develop hybrid cartographic practices and prepare unique, personalized map products. Artificially generated textures can be overlaid with vector data in order to create image maps. The attention-grabbing potential of humanmachine-made maps can be further explored as an interface to more conventional spatiotemporal data representation.

The case study presented a novel approach of utilizing style transfer technique for emotional mapping. This creative human-machine interaction allows artists and cartographers to express their feelings with visual media and utilize it as an input for digital map making. The created paintings and maps serve as a mean of storytelling. Firstly, they form visual metaphors of the city as seen by the participants of the unique mapping event. Secondly, they convey the ideas of unity, contribution or co-creation happening in the city. This case study could be an addition 
to the ongoing discussion of storytelling with maps and further inform the development of visualization tools for expressing subjectivity in space perception.

\section{Acknowledgements}

The research for this paper was financially supported by the Changshu Fengfan Power Equipment Co., Ltd. and assisted by Jiangsu Industrial Technology Research Institute.

We are grateful to the artists from the Autonomous Generative Spirit Programme at the School of Machines, Making \& Make-Believe in Berlin, who provided their artworks, insights and expertise that greatly assisted the research.

\section{References}

Cambria, E., Livingstone, A. and Hussain, A. (2012). The Hourglass of Emotions. In: Esposito A., Esposito A.M., Vinciarelli A., Hoffmann R., Müller V.C. (eds) Cognitive Behavioural Systems. Lecture Notes in Computer Science, vol 7403. Springer, Berlin. DOI: https://doi.org/10.1007/978-3-642-34584-5_11.

Clark, J. (2017). Resurrecting ancient cities with CycleGAN. Available online: https://jackclark.net/2017/06/05/import-ai-issue-45/ (accessed on 25.11.2018).

Field, K. and Demaj D. (2012). Reasserting Design Relevance in Cartography: Some Concepts. The Cartographic Journal, 49:1, 70-76. DOI: 10.1179/0008704112Z.00000000011.

Gatys, L.A., Ecker, A.S. and Bethge, M. (2015). A Neural Algorithm of Artistic Style. arXiv:1508.06576 [cs.CV].

Gatys, L.A., Ecker, A.S. and Bethge M. (2016). Image style transfer using convolutional neural networks. Proceedings of the IEEE Conference on Computer Vision and Pattern Recognition, 27-30 June 2016, Las Vegas. DOI: 10.1109/CVPR.2016.265.

Gombrich, E. (1968). Style. In: Preziosi D. (2009). The Art of Art History: A Critical Anthology. Oxford University Press, Oxford, United Kingdom. ISBN 978-0-19922984-0.

Griffin, A.L. and McQuoid, J. (2012). At the intersection of maps and emotion: The challenge of spatially representing experience. Kartographische Nachrichten, 62:6, 291-299. DOI: 10.19212/kn-2012-06-04.

Isola, P., Zhu, J.Y., Zhou, T. and Efros, A.A. (2017). Image-to-image translation with conditional adversarial networks. arXiv:1611.07004 [cs.CV].

Johnson, J. (2015). Torch implementation of neural style algorithm. Available online: GitHub repository, https://github.com/jcjohnson/neural-style.

Karacan, L., Akata, Z., Erdem, A. and Erdem E. (2018). Manipulating Attributes of Natural Scenes via Hallucination. arXiv:1808.07413 [cs.CV].
Kent, A. (2017). Cartographic Style and the Aesthetic Fix. The Cartographic Journal, 54:1, 1-4. DOI: 10.1080/00087041.2017.1291556.

Klingemann, M. (2018). Old Masters GAN meets SNGAN. Published 18.08.2018, retrieved from https://twitter.com/quasimondo/status/10424444407601 39776s.

Kogan, G., Gambotto, G., Samsen, A., Boleslavský, A., Ferretti, M., Gui, D. and Frei, F. (2016). Invisible cities. Available online: https://opendot.github.io/ml4ainvisible-cities/ (accessed on 25.11.2018).

Machajdik J., Hanbury A. (2010). Affective Image Classification Using Features Inspired by Psychology and Art Theory. Proceedings of the 18th ACM International Conference on Multimedia, 25-29 October 2010, Firenze. DOI: 10.1145/1873951.1873965.

Nold, C. (2009). From device to methodology. In: Emotional Cartography - Technologies of the Self. Available online: http://emotionalcartography.net/ (accessed on 25.11.2018). ISBN 978-0-9557623-1-4.

Robinson, A.C., Demšar, U., Moore, A.B., Buckley, A., Jiang, B., Field, K., Kraak, M-J., Camboim, S.P. and Sluter, C.R. (2017). Geospatial big data and cartography: research challenges and opportunities for making maps that matter. International Journal of Cartography, 3:sup1, 32-60. DOI: 10.1080/23729333.2016.1278151.

Plutchik, R. (1980). Emotion: A Psychoevolutionary Synthesis. Harper and Row, New York. ISBN 0-06045235-8.

Sidonie, C. (2012). Cartographic Styles between traditional and original (towards a cartographic style model). Proceedings of the AutoCarto 2012, The International Research Symposium on Computer-based Cartography, 16-18 September 2012, Columbus, Ohio.

Smith, C. (2016). TensorFlow (Python API) implementation of Neural Style. Available online: GitHub repository, https://github.com/cysmith/neuralstyle-tf.

Thudt, A. F., Perin, C., Willett, W.C. and Carpendale, S. (2017). Subjectivity in personal storytelling with visualization. Information Design Journal, 23(1), 48-64. DOI: $10.175 /$ idj.23.1.07thu.

Tyner, J.A. (1982). Persuasive cartography, Journal of Geography, $\quad 81: 4, \quad 140-144$, DOI: 10.1080/00221348208980868.

You, Q., Luo, J., Jin, H. and Yang, J. (2016). Building a Large Scale Dataset for Image Emotion Recognition: The Fine Print and the Benchmark. Proceedings of the Thirtieth AAAI Conference on Artificial Intelligence, 12-17 February 2016, Phoenix, Arizona, 308-314.

Zhu, J., Park, T., Isola, P. and Efros, A.A. (2017). Unpaired Image-to-Image Translation Using CycleConsistent Adversarial Networks. Proceedings of the IEEE International Conference on Computer Vision (ICCV), 22-29 October 2017, Venice. DOI: 10.1109/ICCV.2017.244. 


\section{Appendix}

\begin{tabular}{|c|c|c|c|c|c|c|c|c|c|c|c|c|c|c|c|c|c|c|c|c|c|c|}
\hline \multirow{5}{*}{$\Xi$} & 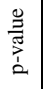 & $\begin{array}{l}\infty \\
\vdots \\
0 \\
0\end{array}$ & $\begin{array}{l}0 \\
\stackrel{0}{0} \\
0 \\
0 \\
0\end{array}$ & 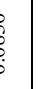 & $\begin{array}{l}\text { वे } \\
\text { ठิ }\end{array}$ & $\stackrel{\Xi}{\bar{\Xi}}$ & ֻัे & & $\begin{array}{l}\text { ⿵人 } \\
\text { o. } \\
0 \\
0\end{array}$ & $\begin{array}{l}\text { ț } \\
\text { } \\
0\end{array}$ & $\begin{array}{l}\text { कृ } \\
\text { ț } \\
0\end{array}$ & ذ્ّ & & $\begin{array}{l}\hat{0} \\
\substack{0 \\
0 \\
0}\end{array}$ & $\begin{array}{l}\text { 守 } \\
\text { 势 }\end{array}$ & \begin{tabular}{c|}
$\vec{b}$ \\
$\substack{0 \\
0 \\
0}$
\end{tabular} & 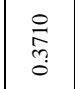 & 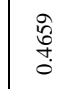 & 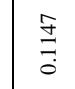 & \begin{tabular}{l} 
fี \\
\multirow{2}{*}{} \\
0
\end{tabular} & & ) \\
\hline & 3 & $\stackrel{\stackrel{f}{f}}{9}$ & 察 & & s. & 先 & ڤั & & in & ప్ & n. & मे & & $\overrightarrow{i n}$ & iิ & 管 & 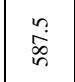 & 隹 & है & $\frac{\infty}{i n}$ & & है \\
\hline & 总 & - 0 & 0 & 0 & 0. & -0 & 0 & & $0-$ & 0.0 & -0 & 0 & & 0. & $\circ 0$ & 0. & 0.0 & 10 & -0 & 0 & $\circ$ & 0 \\
\hline & 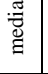 & 00 & & 0. & 00 & -0 & 0 & & $0-$ & 0.0 & -0 & 0 & & 0. & 0.0 & 0.0 & 0 & 100 & $-1-0$ & 0 & & 0 \\
\hline & 䒻 & 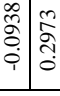 & $\mid \begin{array}{l}0 \\
0 \\
\vdots \\
\vdots \\
\vdots\end{array}$ & & 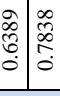 & 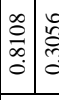 & $\mid \begin{array}{c}\mathfrak{c} \\
\vdots \\
\vdots \\
\vdots \\
\vdots\end{array}$ & & $\begin{array}{lll}f \\
\vdots \\
\vdots \\
\vdots\end{array}$ & 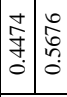 & 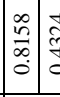 & & & 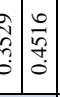 & 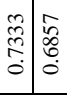 & 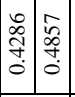 & 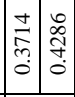 & 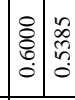 & 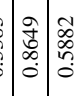 & & 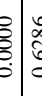 & $\begin{array}{c}\qquad \\
\vdots \\
\vdots \\
\vdots \\
\vdots\end{array}$ \\
\hline \multirow{5}{*}{ 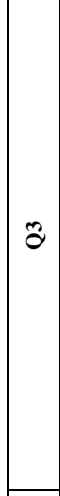 } & 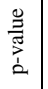 & $\stackrel{\bar{a}}{0}$ & $\begin{array}{l}\text { f } \\
\text { d } \\
\text { d }\end{array}$ & & $\begin{array}{l}\text { o. } \\
\stackrel{\text { Oे }}{0}\end{array}$ & $\begin{array}{l}0 \\
: \\
: \\
0\end{array}$ & तु & & $\begin{array}{l}\text { 话 } \\
\text { đ̃ } \\
0\end{array}$ & $\begin{array}{l}\mathscr{2} \\
\stackrel{2}{0}\end{array}$ & $\stackrel{\frac{t}{m}}{0}$ & 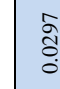 & & $\begin{array}{l}0 \\
\stackrel{0}{n} \\
0 \\
0 \\
0\end{array}$ & $\begin{array}{l}. \\
\stackrel{\circ}{0} \\
0 \\
0\end{array}$ & त्रे & $\begin{array}{l}\vec{b} \\
\stackrel{0}{0}\end{array}$ & 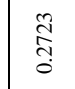 & 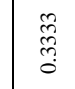 & 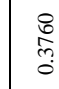 & & 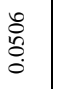 \\
\hline & 3 & $\stackrel{\infty}{\circ}$ & 牟 & t & $\stackrel{m}{n}$ & 悉 & है & & $\begin{array}{l}n \\
\frac{n}{6} \\
\frac{1}{6}\end{array}$ & స్రి & 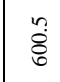 & in & & $\frac{n}{2}$ & $\begin{array}{l}n \\
\mathfrak{a} \\
a\end{array}$ & 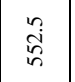 & $\begin{array}{l}n \\
\dot{v} \\
\dot{v}\end{array}$ & 志 & בू. & $\tilde{m}$ & & : \\
\hline & 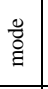 & 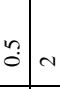 & & - & > - & -- & $\sim$. & & v- & $\begin{array}{lll}4 \\
4\end{array}$ & $\because$ & $\Delta+$ & & $-\sim$ & -- & -4 & --- & $a \mathrm{a}$ & $-=$ & - & - & - \\
\hline & 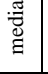 & $\begin{array}{ll}4 \\
8\end{array}$ & &.- & $\sim-$ & -- & $\sim$. & & - & \begin{tabular}{|c|c|}
4 \\
4
\end{tabular} & $\because-$ & 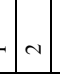 & & $-\sim$ & - & \begin{tabular}{|l|l|} 
& 4 \\
\end{tabular} & -- & $\sqrt{4}$ & $-=$ & - & $-a$ & - \\
\hline & 䒻 & \begin{tabular}{c|cc}
$\frac{m}{\infty}$ \\
$\stackrel{0}{0}$
\end{tabular} & 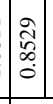 & 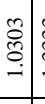 & 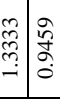 & 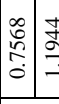 & $\begin{array}{l}0 \\
0 \\
0 \\
0\end{array}$ & & 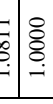 & 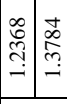 & & & & 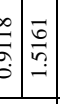 & 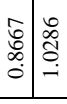 & 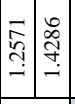 & 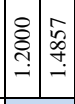 & 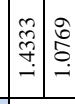 & & & 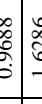 & 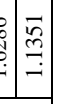 \\
\hline \multirow{5}{*}{ 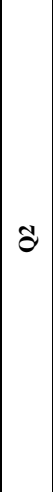 } & 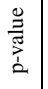 & $\underset{0}{\stackrel{\infty}{+}}$ & $\begin{array}{l}0 \\
\stackrel{0}{0} \\
0 \\
0\end{array}$ & 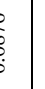 & $\begin{array}{l}\hat{\tilde{o}} \\
\dot{0}\end{array}$ & $\stackrel{0}{\frac{1}{0}}$ & هి & & $\begin{array}{l}\overline{\hat{0}} \\
\stackrel{0}{0}\end{array}$ & 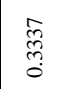 & $\begin{array}{l}\text { ôt } \\
\text { do } \\
\text { d. }\end{array}$ & 홍 & & $\begin{array}{l}8 \\
8 \\
0 \\
0 \\
0\end{array}$ & 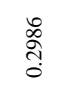 & 항 & $\begin{array}{l}0 \\
\frac{0}{0} \\
0\end{array}$ & 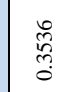 & 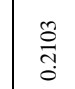 & $\stackrel{+}{\frac{\pi}{3}}$ & & 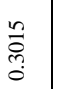 \\
\hline & 3 & $\begin{array}{l}n \\
\stackrel{n}{n} \\
n\end{array}$ & 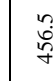 & & $\begin{array}{l}n \\
\text { in } \\
i n\end{array}$ & $\tilde{n}$ & i & & 志 & $\begin{array}{l}n \\
0 \\
0\end{array}$ & 旾 & 要 & & $\begin{array}{l}n \\
\infty \\
\frac{n}{\sigma}\end{array}$ & 品 & 吕 & $\begin{array}{l}n \\
\stackrel{\leftrightarrow}{7} \\
q\end{array}$ & 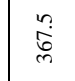 & $\vec{b}$ & \& & & î̀ \\
\hline & 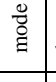 & -4 & & $\sim$ & $\sim-$ & \begin{tabular}{l|c}
$\sim$ & $\sim$ \\
\end{tabular} & - & & -1 & -4 & -- & -- & & $-N$ & - & 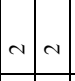 & $-a$ & --- & 4 & - & 1 & $\triangle$ \\
\hline & 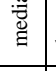 & $\begin{array}{r}-4 \\
-4\end{array}$ & & $\mathrm{~N}$ & $\therefore-$ & 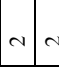 & - & & -4 & $\begin{array}{l}-4 \\
-4\end{array}$ & -3 & $v-$ & & $-\sim$ & -- & \begin{tabular}{|c|c|} 
\\
\end{tabular} & \begin{tabular}{|l|l|} 
& 4 \\
\end{tabular} & --- & $\| \sim$ & - & $-\mathrm{s}$ & ה \\
\hline & 駕 & 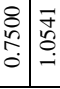 & $\mid \begin{array}{l}0 \\
\vdots \\
0 \\
0 \\
0 \\
0\end{array}$ & בָ & \begin{tabular}{c}
$\vec{c}$ \\
\hdashline
\end{tabular} & \begin{tabular}{l|l}
$\overline{\mathrm{o}}$ & 表 \\
\hdashline
\end{tabular} & $\mid$ & & $\begin{array}{l}f \\
c \\
c\end{array}$ & 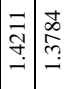 & \begin{tabular}{|l|l|l}
0 \\
0 \\
0 \\
0
\end{tabular} & & & \begin{tabular}{c}
$:$ \\
$:$ \\
\hdashline
\end{tabular} & ষ্ট్: & 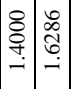 & 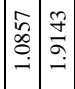 & 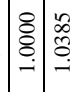 & 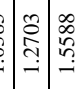 & 辛 & 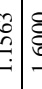 & 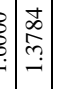 \\
\hline \multirow{5}{*}{$\bar{a}$} & 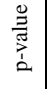 & $\stackrel{\substack{0 \\
0}}{0}$ & $\overline{\bar{f}}$ & 5 & ते & $\begin{array}{l}\text { đ̛ } \\
\text { ป̂. }\end{array}$ & 密 & & 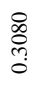 & 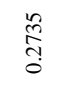 & $\frac{\mathscr{m}}{\tilde{g}}$ & 通 & & \begin{tabular}{l}
$q$ \\
\multirow{2}{*}{} \\
0 \\
0 \\
0
\end{tabular} & $\stackrel{\substack{n \\
\stackrel{g}{g} \\
0}}{0}$ & స్ & $\begin{array}{l}\text { శ్ } \\
\text { ठे. } \\
0\end{array}$ & 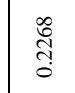 & 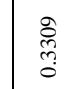 & $\stackrel{\text { 盇 }}{0}$ & & $\frac{n}{n}$ \\
\hline & 3 & în & in & 5 & $\stackrel{\infty}{\Im}$ & $\begin{array}{l}n \\
\infty \\
\vdots \\
\vdots\end{array}$ & ñ. & & $\frac{n}{\vec{A}}$ & $\begin{array}{l}n \\
\text { on } \\
0 \\
0\end{array}$ & : & 帒 & & f & 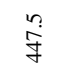 & 芦 & $\frac{n}{f}$ & 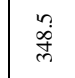 & 今 & $\stackrel{\infty}{\stackrel{\infty}{\sim}}$ & & $\begin{array}{l}n \\
\text { in } \\
\text { in }\end{array}$ \\
\hline & $\begin{array}{l}\stackrel{0}{0} \\
\frac{0}{g}\end{array}$ & -- & & - & $\sim-$ & \begin{tabular}{l|c}
-4 \\
-
\end{tabular} & $\mathrm{A}$. & & $=$ & a - & 0 & $-A$ & & $-\Delta$ & -4 & \begin{tabular}{|c|c|} 
& 4 \\
\end{tabular} & $A$ A & $\mathrm{a} / \mathrm{a}$ & a - & - & $?$ & $\mathrm{~N}$ \\
\hline & 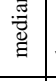 & -- & & - & s- & $-r$ & $\sim \mid$ & & 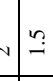 & a - & 0 & $-a$ & & $-\sim$ & -4 & \begin{tabular}{|l|l|} 
& 4 \\
\end{tabular} & \begin{tabular}{|l|l|l|l|l|l|} 
& $A$
\end{tabular} & $\mathrm{~A} / \mathrm{a}$ & N- & - & \multicolumn{2}{|c|}{ 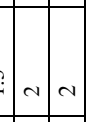 } \\
\hline & 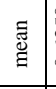 & 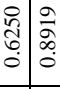 & & 1 & 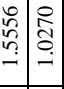 & 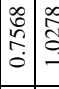 & 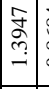 & & 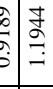 & 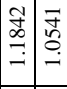 & 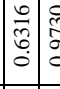 & & $\mid$ & 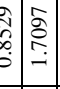 & 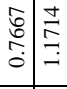 & 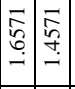 & 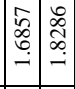 & 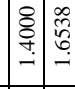 & & $\mid \begin{array}{cc} & \\
\infty & \\
0 & \\
0 & 0\end{array}$ & 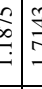 & 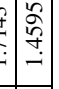 \\
\hline & & $\approx \approx$ & 至 & 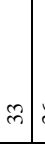 & $\infty \pi$ & 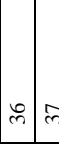 & $\infty$ & & 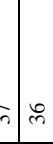 & $\begin{array}{lll}\infty & \tilde{m}\end{array}$ & $\infty$ & $\approx \approx$ & $m$ & $\vec{m}$ & $\ddot{\infty}$ & $\approx \approx$ & $\approx \approx$ & $\infty)_{\infty}$ & $\approx$ & $\approx$ & $\approx$ & $\hat{m}$ \\
\hline $\bar{\omega}$ & & 駦 & & & 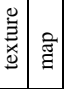 & 畩 & $\mid$ & & 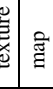 & 总妾 & 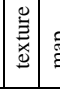 & 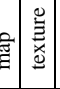 & 嵒 & 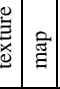 & 总 & 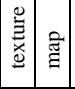 & 胥 & 窇 & 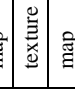 & 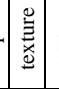 & : & 气̊t: \\
\hline$\frac{E}{4}$ & & $\varangle$ & z & & Ш & $\Phi$ & 0 & & $I$ & - & 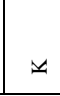 & $\Sigma$ & \pm & z & $\approx$ & 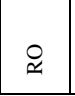 & $\infty$ & $r$ & $\cong$ & $\mathrm{N}$ & & 0 \\
\hline
\end{tabular}

